2018

\title{
Moment Generating Functions of Complementary Exponential-Geometric Distribution Based on k-th Lower Record Values
}

Devendra Kumar

Central University Haryana, Mahendergarh, India, devendrastats@gmail.com

Sanku Dey

King Abdulaziz University, Jeddah, Saudi Arabia, sankud66@gmail.com

Mansoor Rashid Malik

Amity University, Noida, India, mansoormalik884@gmail.com

Fahad M. Al-Aboud

King Abdulaziz University, Jeddah, Saudi Arabia, f_alaboud@hotmail.com

Follow this and additional works at: https:// digitalcommons.wayne.edu/jmasm

Part of the Applied Statistics Commons, Social and Behavioral Sciences Commons, and the Statistical Theory Commons

\section{Recommended Citation}

Kumar, D., Dey, S., Malik, M. R., \& Al-Aboud, F. H. (2018). Moment Generating Functions of Complementary ExponentialGeometric Distribution Based on k-th Lower Record Values. Journal of Modern Applied Statistical Methods, 17(1), eP2479. doi: $10.22237 / \mathrm{jmasm} / 1525133220$ 


\section{Moment Generating Functions of Complementary Exponential- Geometric Distribution Based on k-th Lower Record Values}

\section{Cover Page Footnote}

The authors would like to thank the reviewers and the editors for their comments which helped improve the paper. 


\section{Moment Generating Functions of Complementary Exponential-Geometric Distribution Based on $k$-th Lower Record Values}

\section{Devendra Kumar}

Central University of Haryana

Mahendergarh, India

Mansoor Rashid Malik

Amity University

Noida, India

\author{
Sanku Dey \\ King Abdulaziz University \\ Jeddah, Saudi Arabia \\ Fahad M. Al-Aboud \\ King Abdulaziz University \\ Jeddah, Saudi Arabia
}

The complementary exponential-geometric (CEG) distribution is a useful model for analyzing lifetime data. For this distribution, some recurrence relations satisfied by marginal and joint moment generating functions of $k$-th lower record values were established. They enable the computation of the means, variances, and covariances of $k$-th lower record values for all sample sizes in a simple and efficient recursive manner. Means, variances, and covariances of lower record values were tabulated from samples of sizes up to 10 for various values of the parameters.

Keywords: $\quad k$-th lower record value, marginal and joint moment generating function, recurrence relations, complementary exponential-geometric distribution

\section{Introduction}

Record values and record times are of interest. For example, a meteorologist may deal with upper and lower record temperatures and precipitation levels, or a seismologist may be interested in earthquakes of record magnitude. Several attempts may be made in a sporting event to achieve a record, and records are made only when the attempt is a success. Usually, data on attempts to break records are not available.

doi: 10.22237/jmasm/1525133220 | Accepted: February 8, 2017; Published: June 7, 2018.

Correspondence: Devendra Kumar, devendrastats@gmail.com 


\section{KUMAR ET AL}

Chandler (1952) studied the distributions of lower records, record times, and inter-record times for identically independently distributed (iid) sequences of random variables. This model takes a certain dependence structure into consideration. The life-length distribution of the components in the system may change after each failure of the components. For this type of model, consider the lower record statistics. If various voltages of equipment are considered, only the voltages less than the previous one can be recorded. These recorded voltages are the lower record value sequence. The related literature on record-breaking data and its distributional properties is vast (see, for instance, Chandler, 1952; Resnick, 1973; Shorrock, 1973; Glick, 1978; Nevzorov, 1987; Ahsanullah, 1995; Balakrishnan \& Ahsanullah, 1993, 1994; Grunzień \& Szynal, 1997; Arnold, Balakrishnan, \& Nagaraja, 1992, 1998; Pawlas \& Szynal, 1999; Kumar, 2012, 2015 , 2016; Kumar \& Kulshrestha, 2013).

A random variable $X$ is said to have complementary exponential-geometric distribution (see Louzada, Roman, \& Cancho, 2011) if its pdf is of the form

$$
\mathrm{f}(x)=\frac{\theta e^{-x / \beta}}{\beta\left[e^{-x / \beta}(1-\theta)+\theta\right]^{2}}, \quad x>0, \beta>0,0<\theta<1
$$

and the corresponding cdf is

$$
\mathrm{F}(x)=\frac{\theta\left(1-e^{-x / \beta}\right)}{\left[e^{-x / \beta}(1-\theta)+\theta\right]}, \quad x>0, \beta>0,0<\theta<1
$$

Note that the complementary exponential-geometric distribution defined in (1) can expressed as

$$
\mathrm{f}(x)= \begin{cases}-(1-\theta)[1-\mathrm{F}(x)]^{2}+[1-\mathrm{F}(x)] & (*) \\ -(1-\theta)[\mathrm{F}(x)]^{2}+(1-2 \theta)[\mathrm{F}(x)]+\theta & (* *)\end{cases}
$$

Here $\beta$ is the scale parameter and $\theta$ is the shape parameter. The distribution exhibits decreasing and unimodal pdfs and increasing hazard rates.

The concept of recurrence is useful in reducing the number of operations necessary to obtain a general form for the function under consideration. It is also 


\section{MOMENT GENERATING FUNCTIONS OF CEG DISTRIBUTION}

used in characterizing the distributions, permitting the identification of population distribution from the properties of the sample.

One of the motivations here is the relative paucity of work concerning marginal and joint moment generating functions of $k$-th lower record values. The objective is to establish an explicit expression and recurrence relations for marginal and joint moment generating functions of $k$-th lower record values in a simple recursive manner. The so-obtained relationships enable us to compute all the moments of lower record values through using some mathematical software (e.g., Mathematica, Maple).

\section{Record Values and Preliminaries}

Let $\left\{X_{n}, n \geq 1\right\}$ be a sequence of iid random variables with cdf $\mathrm{F}(x)$ and $\operatorname{pdf} \mathrm{f}(x)$. The $j^{\text {th }}$ order statistics of a sample $\left(X_{1}, X_{2}, \ldots, X_{n}\right)$ is denoted by $X_{j: n}$. For a fixed $k \geq 1$, define the sequence $\left\{\mathrm{L}^{(k)}(n), n \geq 1\right\}$ of $k$-th lower record times of $X_{1}, X_{2}, \ldots$ as follows:

$$
\begin{aligned}
\mathrm{L}^{(k)}(1) & =1 \\
\mathrm{~L}^{(k)}(n+1) & =\min \left\{j>\mathrm{L}^{(k)}(n), X_{k: \mathrm{L}^{(k)}(n)+k-1}>X_{k: j+k-1}\right\}
\end{aligned}
$$

The sequences $\left\{Z_{n}^{(k)}, n \geq 1\right\}$ with $Z_{n}^{(k)}=X_{k: \mathrm{L}^{(k)}(n)+k-1}, n=1,2, \ldots$ are called the sequences of $k$-th lower record values of $\left\{X_{n}, n \geq 1\right\}$. For convenience, take $Z_{0}^{(k)}=0$. Note that $k=1, Y_{1}^{(1)}=X_{\mathrm{L}(n)}, n \geq 1$, i.e. record values of $\left\{X_{n}, n \geq 1\right\}$. The joint pdf of $k$-th lower record values $Z_{1}^{(k)}, Z_{2}^{(k)}, \ldots, Z_{n}^{(k)}$ can be given as the joint pdf of $k$-th lower record values of $\left\{-X_{n}, n \geq 1\right\}$ is given by

$$
\mathrm{f}_{Z_{1}^{(k)}, Z_{2}^{(k)}, \ldots, Z_{n}^{(k)}}\left(z_{1}, z_{2}, \ldots, z_{n}\right)=k^{n}\left(\prod_{i=1}^{n-1} \frac{\mathrm{f}\left(z_{i}\right)}{\mathrm{F}\left(z_{i}\right)}\right)\left[\mathrm{F}\left(z_{n}\right)\right]^{k-1} \mathrm{f}\left(z_{n}\right), \quad z_{1}>z_{2}>\ldots>z_{n}
$$

In view of above equation, the marginal pdf of $X_{\mathrm{L}(n)}^{(k)}, n \geq 1$, is given by

$$
\mathrm{f}_{X_{\llcorner(n)}^{(k)}}(x)=\frac{k^{n}}{(n-1) !}[-\ln (\mathrm{F}(x))]^{n-1}[\mathrm{~F}(x)]^{k-1} \mathrm{f}(x)
$$




\section{KUMAR ET AL}

and the joint pdf of $X_{\mathrm{L}(m)}^{(k)}$ and $X_{\mathrm{L}(n)}^{(k)}, 1 \leq m<n, n>2$, is given by

$$
\begin{aligned}
\mathrm{f}_{X_{\mathfrak{L}^{(k)}(k)}^{(k)}, x_{\llcorner(n)}^{(k)}}(x, y)=\frac{k^{n}}{(m-1) !(n-m-1) !}[-\ln (\mathrm{F}(x))]^{m-1} \\
\quad \times[-\ln (\mathrm{F}(y))+\ln (\mathrm{F}(x))]^{n-m-1}[\mathrm{~F}(y)]^{k-1} \frac{\mathrm{f}(x)}{\mathrm{F}(x)} \mathrm{f}(y)
\end{aligned}
$$

for $x>y$. Let $X_{1}, X_{2}, \ldots, X_{n}$ be a random sample of the complementary exponentialgeometric distribution with pdf and cdf as in (1) and (2), respectively, and let $X_{\mathrm{L}(1)}$, $X_{\mathrm{L}(2),}, \ldots, X_{\mathrm{L}(n)}$ be the first $n$ lower record values obtained from this sample. Denote the marginal moment generating functions of $X_{\mathrm{L}(n): k}$ by $\mathrm{M}_{X_{\mathrm{L}(n) k}}(t)$ and its $j^{\text {th }}$

derivative by $\mathbf{M}_{X_{\llcorner(n) k}}^{(j)}(t)$. Similarly, let $\mathbf{M}_{X_{\llcorner(m, n) k}}\left(t_{1}, t_{2}\right)$ and $\mathbf{M}_{X_{\lfloor(m, n) k}}^{(i, j)}\left(t_{1}, t_{2}\right)$ denote the joint moment generating functions of $X_{\mathrm{L}(m): k}$ and $X_{\mathrm{L}(n): k}$ and its $(i, j)^{\text {th }}$ partial derivatives with respect to $t_{1}$ and $t_{2}$, respectively.

\section{Relations for Marginal Moment Generating Function}

Establish the explicit expression and recurrence relations for marginal moment generating functions of $k$-th lower record values from complementary exponentialgeometric distribution as follows:

Theorem 1. For the distribution given in (3), a positive integer $k \geq 1$, and for $n \geq 1$,

$$
\mathbf{M}_{X_{\llcorner(n) k}}(t)=k^{n} \sum_{p=0}^{\infty} \sum_{l=0}^{\infty} \frac{\Gamma(\beta t+1)}{\Gamma(\beta t+1-p)} \frac{(p)_{l}}{\theta^{p} p ! l !(k+p+l)^{n}}, \quad t \neq 0
$$

where

$$
(t)_{l}= \begin{cases}t(t+1) \cdots(t+p-1), & p=1,2, \ldots \\ 1, & p=0\end{cases}
$$

\section{Proof. $\quad$ From (3)}




\section{MOMENT GENERATING FUNCTIONS OF CEG DISTRIBUTION}

$$
\begin{aligned}
\mathrm{M}_{X_{\mathrm{L}(n) k}}(t) & =\frac{k^{n}}{(n-1) !} \int_{0}^{\infty} e^{t x}[-\ln (\mathrm{F}(x))]^{n-1}[\mathrm{~F}(x)]^{k-1} \mathrm{f}(x) d x \\
& =\frac{k^{n}}{(n-1) !} \int_{0}^{1}\left[1+\frac{z}{\theta(1-z)}\right]^{\beta t} z^{k-1}[-\ln z]^{n-1} d z \\
& =\frac{k^{n}}{(n-1) !} \sum_{p=0}^{\infty} \frac{\Gamma(\beta t+1)}{\theta^{p} p ! \Gamma(\beta t+1-p)} \int_{0}^{1} z^{p+k-1}(1-z)^{-p}[-\ln z]^{n-1} d z \\
& =\frac{k^{n}}{(n-1) !} \sum_{p=0}^{\infty} \sum_{l=0}^{\infty} \frac{\Gamma(\beta t+1)(p)_{l}}{\theta^{p} p ! l ! \Gamma(\beta t+1-p)} \int_{0}^{1} z^{p+k+l-1}[-\ln z]^{n-1} d z \\
& =\frac{k^{n}}{(n-1) !} \sum_{p=0}^{\infty} \sum_{l=0}^{\infty} \frac{\Gamma(\beta t+1)(p)_{l}}{\theta^{p} p ! l ! \Gamma(\beta t+1-p)} \int_{0}^{\infty} e^{-(p+k+l) u} u^{n-1} d u
\end{aligned}
$$

where $z=\mathrm{F}(x)$ and $u=-\ln z$. The result follows by the definition of complete gamma distribution.

Remark 1. Setting $k=1$ in (5), deduce the explicit expression for marginal moment generating functions of lower record values from the complementary exponential-geometric distribution.

Recurrence relations for marginal moment generating functions of $k$-th lower record values from the cdf (2) can be derived in the following theorem:

Theorem 2. For a positive integer $k>1$, and for $n \geq 1$ and $j=1,2, \ldots$,

$$
\begin{aligned}
\mathbf{M}_{X_{\mathrm{L}(n) k+1}}^{(j)}(t)= & \left.\frac{k+1}{k-1}\right)^{n-1} \frac{\theta}{1-\theta}\left[\mathbf{M}_{X_{\mathrm{L}(n) k-1}}^{(j)}(t)-\mathbf{M}_{X_{\mathrm{L}(n-1) k-1}}^{(j)}(t)\right] \\
& +\left(\frac{k+1}{k}\right)^{n-1} \frac{(1-2 \theta)}{1-\theta}\left[\mathbf{M}_{X_{\mathrm{L}(n) k}}^{(j)}(t)-\mathbf{M}_{X_{\mathrm{L}(n-1) k}}^{(j)}(t)\right] \\
& -\frac{(k+1)^{n-1}}{k^{n}(1-\theta)}\left[t \mathbf{M}_{X_{\mathrm{L}(n) \cdot k}}^{(j)}(t)+j \mathbf{M}_{X_{\mathrm{L}(n-1) k}}^{(j)}(t)\right]+\mathbf{M}_{X_{\mathrm{L}(n-1) k+1}}^{(j)}(t)
\end{aligned}
$$

Proof. $\quad$ From (*), 


\section{KUMAR ET AL}

$$
\begin{aligned}
\mathrm{M}_{X_{L_{(n)) k}}}(t)= & \frac{k^{n}}{(n-1) !} \int_{0}^{\infty} e^{t x}[\mathrm{~F}(x)]^{k-1}[-\ln (\mathrm{F}(x))]^{n-1} \mathrm{f}(x) \\
& \times\left[-(1-\theta)\{1-\mathrm{F}(x)\}^{2}+\{1-\mathrm{F}(x)\}\right] d x \\
= & -\frac{k^{n}(1-\theta)}{(n-1) !}\left[I_{1}+I_{2}-2 I_{3}\right]+\frac{k^{n}}{(n-1) !}\left[I_{1}-I_{3}\right]
\end{aligned}
$$

where

$$
\begin{aligned}
& I_{1}=\int_{0}^{\infty} e^{t x}[\mathrm{~F}(x)]^{k-1}[-\ln (\mathrm{F}(x))]^{n-1} d x \\
& I_{2}=\int_{0}^{\infty} e^{t x}[\mathrm{~F}(x)]^{k+1}[-\ln (\mathrm{F}(x))]^{n-1} d x \\
& I_{3}=\int_{0}^{\infty} e^{t x}[\mathrm{~F}(x)]^{k}[-\ln (\mathrm{F}(x))]^{n-1} d x
\end{aligned}
$$

By integrating by parts,

$$
\begin{aligned}
& I_{1}=\frac{(n-1) !}{t(k-1)^{n-1}}\left[\mathrm{M}_{X_{\lfloor(n-1)(k-1}}(t)-\mathrm{M}_{X_{\lfloor(n) k-1}}(t)\right] \\
& I_{2}=\frac{(n-1) !}{t(k+1)^{n-1}}\left[\mathrm{M}_{X_{\lfloor(n-1) k+1}}(t)-\mathrm{M}_{X_{\lfloor(n) k+1}}(t)\right]
\end{aligned}
$$

and

$$
I_{3}=\frac{(n-1) !}{t k^{n-1}}\left[\mathrm{M}_{X_{\lfloor(n-1) k}}(t)-\mathrm{M}_{X_{\lfloor(n) k}}(t)\right]
$$

Combining (7) and (8)-(10), 


\section{MOMENT GENERATING FUNCTIONS OF CEG DISTRIBUTION}

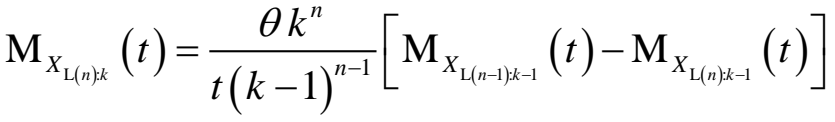

$$
\begin{aligned}
& -\frac{(1-\theta) k^{n}}{t(k-1)^{n-1}}\left[\mathrm{M}_{X_{\mathrm{L}_{(n-1) k+1}}}(t)-\mathrm{M}_{X_{\mathrm{L}^{(n) k+1}}}(t)\right] \\
& +\frac{(1-2 \theta) k}{t}\left[\mathrm{M}_{X_{L^{(n-1) ! k}}}(t)-\mathrm{M}_{X_{\lfloor(n) k}}(t)\right]
\end{aligned}
$$

Now, differentiating both the sides of (11) $j$ times with respect to $t$,

$$
\begin{aligned}
& \left(\frac{1-\theta}{(k+1)^{n-1}}\right) \mathbf{M}_{X_{L_{(n) k+1}}^{(j)}}(t) \\
& =\frac{\theta}{(k-1)}\left[\mathbf{M}_{X_{\mathrm{L}(n) k-1}}^{(j)}(t)-\mathbf{M}_{X_{L_{(n-1) k-1}}}^{(j)}(t)\right] \\
& -\frac{1}{k^{n}}\left[t \mathrm{M}_{X_{\lfloor(n) k}}^{(j)}(t)+j \mathrm{M}_{X_{\lfloor(n) k}}^{(j-1)}(t)\right]+\left(\frac{1-\theta}{(k+1)^{n-1}}\right) \mathbf{M}_{X_{\lfloor(n-1) k+1}}^{(j)}(t) \\
& +\frac{1-2 \theta}{k^{n-1}}\left[\mathrm{M}_{X_{\llcorner(n) k}}^{(j)}(t)-\mathrm{M}_{X_{\lfloor(n-1) k}}^{(j)}(t)\right]
\end{aligned}
$$

The proof is complete.

Similarly, by using the relation in equation $(* *)$ and proceeding as above, obtain the following result:

Theorem 3. For a positive integer $k>1$, and for $n \geq 1$ and $j=1,2, \ldots$,

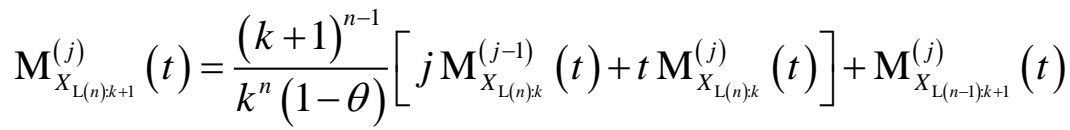

$$
\begin{aligned}
& -\frac{(1-2 \theta)}{(1-\theta)}\left(\frac{k+1}{k}\right)^{n-1}\left[\mathbf{M}_{X_{\llcorner(n-1) k}}^{(j)}(t)-t \mathbf{M}_{X_{\lfloor(n) k}}^{(j)}(t)\right] \\
& -\frac{\theta}{(1-\theta)}\left(\frac{k+1}{k-1}\right)^{n-1}\left[\mathbf{M}_{X_{L^{(n-1) k k-1}}}^{(j)}(t)-\mathbf{M}_{X_{L^{\prime}(n) k-1}}^{(j)}(t)\right]
\end{aligned}
$$

Proof. $\quad$ Similar to the proof of Theorem 2. 


\section{KUMAR ET AL}

By differentiating both sides of equations (6) and (13) with respect to $t$ and then setting $t=0$, we can obtain the recurrence relations for single moment of $k$-th lower record values from the complementary exponential-geometric distribution.

$$
\begin{aligned}
\mathrm{E}\left[X_{\mathrm{L}(n): k+1}^{(j)}\right]= & \frac{\left.(k+1)^{n-1}\right)\left\{\frac{\theta}{1-\theta} \mathrm{E}\left[X_{\mathrm{L}(n): k-1}^{(j)}\right]-\mathrm{E}\left[X_{\mathrm{L}(n-1): k-1}^{(j)}\right]\right\}}{}+\frac{(1-2 \theta)}{k^{n-1}}\left\{\mathrm{E}\left[X_{\mathrm{L}(n): k}^{(j)}\right]-\mathrm{E}\left[X_{\mathrm{L}(n-1): k}^{(j)}\right]\right\} \\
& -\frac{j}{k^{n}} \mathrm{E}\left[X_{L(n): k}^{(j-1)}\right]+\frac{(1-\theta)}{(k+1)^{n-1}} \mathrm{E}\left[X_{\mathrm{L}(n-1): k+1}^{(j)}\right]
\end{aligned}
$$

and

$$
\begin{aligned}
\mathrm{E}\left[X_{\mathrm{L}(n): k+1}^{(j)}\right] & \\
= & \frac{j(k+1)^{n-1}}{k^{n}(1-\theta)} \mathrm{E}\left[X_{\mathrm{L}(n): k}^{(j-1)}\right]-\left(\frac{1-2 \theta}{1-\theta}\right)\left(\frac{k+1}{k}\right)^{n-1}\left\{\mathrm{E}\left[X_{\mathrm{L}(n-1): k}^{(j)}\right]-\mathrm{E}\left[X_{\mathrm{L}(n): k}^{(j)}\right]\right\} \\
& -\frac{\theta}{1-\theta}\left(\frac{k+1}{k-1}\right)^{n-1}\left\{\mathrm{E}\left[X_{\mathrm{L}(n-1): k-1}^{(j)}\right]-\mathrm{E}\left[X_{\mathrm{L}(n): k-1}^{(j)}\right]\right\}+\mathrm{E}\left[X_{\mathrm{L}(n-1): k+1}^{(j)}\right]
\end{aligned}
$$

Remark 2. Setting $k=1$ in (6) and (13), deduce the recurrence relations for marginal moment generating functions of lower record values from the complementary exponential-geometric distribution.

\section{Relations for Joint Moment Generating Function}

Next, establish the explicit and recurrence relations for joint moment generating function of $k$-th lower record values from complementary exponential-geometric distribution.

Theorem 4. For a positive integer $k>1$, and for $n \geq 1$, 


$$
\begin{aligned}
\mathrm{M}_{X_{\mathrm{L}(m, n)}}\left(t_{1}, t_{2}\right)= & k^{n} \sum_{p=0}^{\infty} \sum_{l=0}^{\infty} \sum_{q=0}^{\infty} \sum_{r=0}^{\infty} \frac{\Gamma\left(\beta t_{1}+1\right) \Gamma\left(\beta t_{2}+1\right)(p)_{l}}{l ! p ! \theta^{p+q} \Gamma\left(\beta t_{1}+1-q\right) \Gamma\left(\beta t_{2}+1-p\right)} \\
& \times \frac{(q)_{r}}{r ! q !(p+l+k)^{n-m}(p+l+q+r+k)^{m}}
\end{aligned}
$$

Proof. $\quad$ From (4),

$$
\begin{aligned}
& \mathrm{M}_{X_{\mathrm{L}(m, n)}}\left(t_{1}, t_{2}\right)=\frac{k^{n}}{(m-1) !(n-m-1) !} \int_{0}^{\infty} \int_{0}^{x} e^{t_{1} x+t_{2} y}[-\ln (\mathrm{F}(x))]^{m-1} \frac{\mathrm{f}(x)}{\mathrm{F}(x)} \\
& \times[-\ln (\mathrm{F}(y))+\ln (\mathrm{F}(x))]^{n-m-1}[\mathrm{~F}(y)]^{k-1} \mathrm{f}(y) d y d x \\
& =\frac{k^{n}}{(m-1) !(n-m-1) !} \int_{0}^{\infty} e^{t_{1} x}[-\ln (\mathrm{F}(x))]^{m-1} \frac{\mathrm{f}(x)}{\mathrm{F}(x)} \\
& \times\left(\int_{0}^{\infty}\left[1+\frac{\mathrm{F}(x) e^{-w}}{\theta\left(1-\mathrm{F}(x) e^{-w}\right)}\right]^{\beta t_{2}} w^{n-m-1}\left[\mathrm{~F}(x) e^{-w}\right]^{k} d w\right) d x \\
& =\frac{k^{n}}{(m-1) !} \sum_{p=0}^{\infty} \sum_{l=0}^{\infty} \frac{\Gamma\left(\beta t_{2}+1\right)(p)_{l}}{l ! p ! \theta^{p} \Gamma\left(\beta t_{2}+1-p\right)(p+k+l)^{n-m}} \\
& \times \int_{0}^{\infty} e^{t_{1} x}[\mathrm{~F}(x)]^{p+k+l-1}[-\ln (\mathrm{F}(x))]^{m-1} \mathrm{f}(x) d x \\
& =\frac{k^{n}}{(m-1) !} \sum_{p=0}^{\infty} \sum_{l=0}^{\infty} \sum_{q=0}^{\infty} \sum_{r=0}^{\infty} \frac{\Gamma\left(\beta t_{2}+1\right)(p)_{l}}{l ! p ! \theta^{p} \Gamma\left(\beta t_{2}+1-p\right)(p+k+l)^{n-m}} \\
& \times \frac{\Gamma\left(\beta t_{1}+1\right)(q)_{r}}{q ! r ! \theta^{q} \Gamma\left(\beta t_{1}+1-q\right)} \int_{0}^{\infty} e^{-u(k+p+l+r+k)} u^{m-1} d u
\end{aligned}
$$

where $w=\ln \mathrm{F}(x)-\ln \mathrm{F}(y)$ and $u=-\ln \mathrm{F}(x)$. The result follows by the definition of complete gamma distribution function.

Remark 3. Setting $k=1$ in (14), deduce the explicit expression for joint moment generating function of lower record value from the complementary exponential-geometric distribution.

Theorem 5. For a positive integer $1 \leq m \leq n-2$ and $i, j=1,2, \ldots$, 


$$
\begin{aligned}
\mathbf{M}_{X_{\mathrm{L}(m, n) k}}^{(i, j)}\left(t_{1}, t_{2}\right) & \frac{1}{(1+k(1-2 \theta)) t_{2}}\left[k \theta ( \frac { k } { k - 1 } ) ^ { n - 1 } \left[\mathbf{M}_{X_{\mathrm{L}(m, n-1) k k-1}}^{(i, j)}\left(t_{1}, t_{2}\right)\right.\right. \\
& \left.-t_{2} \mathbf{M}_{X_{\mathrm{L}(m, n) k-1}}^{(i, j)}\left(t_{1}, t_{2}\right)-j \mathbf{M}_{X_{\mathrm{L}(m, n) k k-1}}^{(i, j-1)}\left(t_{1}, t_{2}\right)\right]+(1-\theta) k\left(\frac{k}{k-1}\right)^{n-1} \\
& \times\left[t_{2} \mathbf{M}_{X_{\mathrm{L}(m, n) k+1}}^{(i, j)}\left(t_{1}, t_{2}\right)-\mathbf{M}_{X_{\mathrm{L}(m, n-1) k+1}}^{(i, j)}\left(t_{1}, t_{2}\right)+j \mathbf{M}_{X_{\mathrm{L}(m, n) k+1}}^{(i, j-1)}\left(t_{1}, t_{2}\right)\right] \\
& \left.-(j+j k(1-2 \theta)) \mathbf{M}_{X_{\mathrm{L}(m, n) k \cdot k}}^{(i, j-1)}\left(t_{1}, t_{2}\right)+(1-2 \theta) \mathbf{M}_{X_{\mathrm{L}(m, n-1) k k}}^{(i, j)}\left(t_{1}, t_{2}\right)\right]
\end{aligned}
$$

Proof. $\quad$ From (4), for $1 \leq m \leq n-2$ and $i, j=1,2, \ldots$,

$$
\begin{aligned}
\mathrm{M}_{X_{\mathrm{L}(m, n)}}\left(t_{1}, t_{2}\right)= & \frac{k^{n}}{(m-1) !(n-m-1) !} \int_{0}^{\infty} \int_{0}^{x} e^{t_{1} x+t_{2} y}[-\ln (\mathrm{F}(x))]^{m-1} \frac{\mathrm{f}(x)}{\mathrm{F}(x)} \\
& \times[-\ln (\mathrm{F}(y))+\ln (\mathrm{F}(x))]^{n-m-1}[\mathrm{~F}(y)]^{k-1} \\
& \times\left[\theta+(1-2 \theta) \mathrm{F}(y)-(1-\theta)[\mathrm{F}(y)]^{2}\right] d y d x \\
= & I_{5}+I_{6}+I_{7}
\end{aligned}
$$

where

$$
\begin{aligned}
I_{5}= & \frac{\theta k^{n}}{(m-1) !(n-m-1) !} \int_{0}^{\infty} \int_{0}^{x} e^{t_{1} x+t_{2} y}[-\ln (\mathrm{F}(x))]^{m-1} \frac{\mathrm{f}(x)}{\mathrm{F}(x)} \\
& \times[-\ln (\mathrm{F}(y))+\ln (\mathrm{F}(x))]^{n-m-1}[\mathrm{~F}(y)]^{k-1} d y d x \\
I_{6}= & \frac{(1-2 \theta) k^{n}}{(m-1) !(n-m-1) !} \int_{0}^{\infty} \int_{0}^{x} e^{t_{1} x+t_{2} y}[-\ln (\mathrm{F}(x))]^{m-1} \frac{\mathrm{f}(x)}{\mathrm{F}(x)} \\
& \times[-\ln (\mathrm{F}(y))+\ln (\mathrm{F}(x))]^{n-m-1}[\mathrm{~F}(y)]^{k} d y d x \\
I_{7}= & \frac{(1-\theta) k^{n}}{(m-1) !(n-m-1) !} \int_{0}^{\infty} \int_{0}^{x} e^{t_{1} x+t_{2} y}[-\ln (\mathrm{F}(x))]^{m-1} \frac{\mathrm{f}(x)}{\mathrm{F}(x)} \\
& \times[-\ln (\mathrm{F}(y))+\ln (\mathrm{F}(x))]^{n-m-1}[\mathrm{~F}(y)]^{k+1} d y d x
\end{aligned}
$$




\section{MOMENT GENERATING FUNCTIONS OF CEG DISTRIBUTION}

By integrating by parts,

$$
\begin{aligned}
I_{5}= & \frac{\theta(n-m-1) k^{n}}{t_{2}(m-1) !(n-m-1) !} \int_{0}^{\infty} \int_{0}^{x} e^{t_{1} x+t_{2} y}[-\ln (\mathrm{F}(x))]^{m-1} \frac{\mathrm{f}(x)}{\mathrm{F}(x)} \\
& \times[-\ln (\mathrm{F}(y))+\ln (\mathrm{F}(x))]^{n-m-2}[\mathrm{~F}(y)]^{k-2} \mathrm{f}(y) d y d x \\
& -\frac{\theta(k-1) k^{n}}{t_{2}(m-1) !(n-m-1) !} \int_{0}^{\infty} \int_{0}^{x} e^{t_{1} x+t_{2} y}[-\ln (\mathrm{F}(x))]^{m-1} \frac{\mathrm{f}(x)}{\mathrm{F}(x)} \\
& \times[-\ln (\mathrm{F}(y))+\ln (\mathrm{F}(x))]^{n-m-1}[\mathrm{~F}(y)]^{k-2} \mathrm{f}(y) d y d x \\
I_{6}= & \frac{(1-2 \theta)(n-m-1) k^{n}}{t_{2}(m-1) !(n-m-1) !} \int_{0}^{\infty} \int_{0}^{x} e^{t_{1} x+t_{2} y}[-\ln (\mathrm{F}(x))]^{m-1} \frac{\mathrm{f}(x)}{\mathrm{F}(x)} \\
& \times[-\ln (\mathrm{F}(y))+\ln (\mathrm{F}(x))]^{n-m-2}[\mathrm{~F}(y)]^{k-1} \mathrm{f}(y) d y d x \\
& -\frac{(1-2 \theta) k^{n}}{t_{2}(m-1) !(n-m-1) !} \int_{0}^{\infty} \int_{0}^{x} e^{t_{1} x+t_{2} y}[-\ln (\mathrm{F}(x))]^{m-1} \frac{\mathrm{f}(x)}{\mathrm{F}(x)} \\
& \times[-\ln (\mathrm{F}(y))+\ln (\mathrm{F}(x))]^{n-m-1}[\mathrm{~F}(y)]^{k-1} \mathrm{f}(y) d y d x
\end{aligned}
$$

and

$$
\begin{aligned}
I_{7}= & \frac{(1-\theta)(n-m-1) k^{n}}{t_{2}(m-1) !(n-m-1) !} \int_{0}^{\infty} \int_{0}^{x} e^{t_{1} x+t_{2} y}[-\ln (\mathrm{F}(x))]^{m-1} \frac{\mathrm{f}(x)}{\mathrm{F}(x)} \\
& \times[-\ln (\mathrm{F}(y))+\ln (\mathrm{F}(x))]^{n-m-2}[\mathrm{~F}(y)]^{k} \mathrm{f}(y) d y d x \\
& -\frac{(1-\theta) k^{n}}{t_{2}(m-1) !(n-m-1) !} \int_{0}^{\infty} \int_{0}^{x} e^{t_{1} x+t_{2} y}[-\ln (\mathrm{F}(x))]^{m-1} \frac{\mathrm{f}(x)}{\mathrm{F}(x)} \\
& \times[-\ln (\mathrm{F}(y))+\ln (\mathrm{F}(x))]^{n-m-1}[\mathrm{~F}(y)]^{k} \mathrm{f}(y) d y d x
\end{aligned}
$$

Combining equations (16) and (17)-(19), 


\section{KUMAR ET AL}

$$
\begin{aligned}
& \mathrm{M}_{X_{\mathrm{L}(m, n): k}}\left(t_{1}, t_{2}\right) \\
& =\frac{k \theta}{t_{2}}\left(\frac{k}{k-1}\right)^{n-1}\left[\mathrm{M}_{X_{\lfloor(m, n-1) ! k}}\left(t_{1}, t_{2}\right)-t_{2} \mathrm{M}_{X_{\llcorner(m, n) k)<-1}}\left(t_{1}, t_{2}\right)\right] \\
& +\frac{(1-2 \theta)}{t_{2}}\left[\mathrm{M}_{X_{\mathrm{L}_{(m, n-1) ! k}}}\left(t_{1}, t_{2}\right)-t_{2} k \mathrm{M}_{X_{\lfloor(m, n) \cdot k}}\left(t_{1}, t_{2}\right)\right] \\
& -\frac{(1-\theta) k}{t_{2}}\left(\frac{k}{k+1}\right)^{n-1}\left[\mathrm{M}_{X_{\lfloor(m, n-1) k+1}}\left(t_{1}, t_{2}\right)-t_{2} \mathrm{M}_{X_{\llcorner(m, n) k+1}}\left(t_{1}, t_{2}\right)\right]
\end{aligned}
$$

Differentiating both the sides of (20) $i$ times with respect to $t_{1}$ and then $j$ times with respect to $t_{2}$,

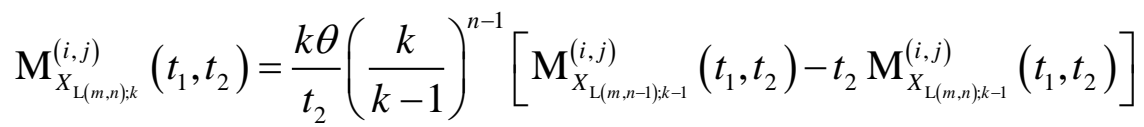

$$
\begin{aligned}
& +\frac{(1-2 \theta)}{t_{2}}\left[\mathbf{M}_{X_{\mathrm{L}(m, n-1) k}}^{(i, j)}\left(t_{1}, t_{2}\right)-t_{2} k \mathbf{M}_{X_{\lfloor(m, n) k}}^{(i, j)}\left(t_{1}, t_{2}\right)\right]
\end{aligned}
$$

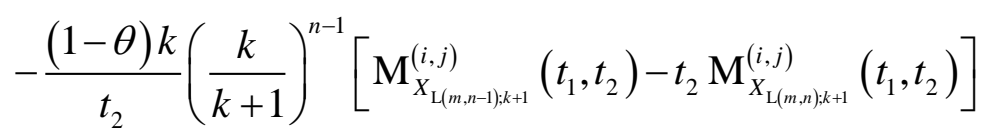

$$
\begin{aligned}
& -\frac{j k \theta}{t_{2}}\left(\frac{k}{k-1}\right)^{n-1} \mathbf{M}_{X_{\lfloor(m, n) k-1}}^{(i, j-1)}\left(t_{1}, t_{2}\right)-k(1-2 \theta) j \mathbf{M}_{X_{\llcorner(m, n) k \cdot k}}^{(i, j-1)}\left(t_{1}, t_{2}\right) \\
& +\frac{k(1-\theta)}{t_{2}}\left(\frac{k}{k+1}\right)^{n-1} j \mathrm{M}_{X_{\lfloor(m, n) k+1}}^{(i, j-1)}\left(t_{1}, t_{2}\right)-j \mathrm{M}_{X_{\llcorner(m, n) k)}}^{(i, j-1)}\left(t_{1}, t_{2}\right)
\end{aligned}
$$

The proof is complete.

Similarly, by using the relation in equation $(*)$ and proceeding as above, obtain the following result:

Theorem 6. For a positive integer $1 \leq m \leq n-2$ and $i, j=1,2, \ldots$, 


\section{MOMENT GENERATING FUNCTIONS OF CEG DISTRIBUTION}

$$
\begin{aligned}
& \mathbf{M}_{X_{\lfloor(m, n) k}}^{(i, j)}\left(t_{1}, t_{2}\right) \\
& =\frac{1}{t_{2}(1+k(1-2 \theta))}\left[\frac{k^{n} \theta}{(k-1)^{n-1}} \mathbf{M}_{X_{L_{(m, n-1) k-1}}^{(i, j)}}\left(t_{1}, t_{2}\right)-\frac{k^{n} \theta}{(k-1)^{n-2}}\right. \\
& \times\left(t_{2} \mathbf{M}_{X_{\mathrm{L}(m, n) k)-1}}^{(i, j)}\left(t_{1}, t_{2}\right)+j \mathbf{M}_{X_{\mathrm{L}(m, n) k<-1}}^{(i, j-1)}\left(t_{1}, t_{2}\right)\right)-j \mathbf{M}_{X_{\lfloor(m, n) k}}^{(i, j-1)}\left(t_{1}, t_{2}\right) \\
& +(1-2 \theta) \mathbf{M}_{X_{\lfloor(m, n-1) k}}^{(i, j)}\left(t_{1}, t_{2}\right)-(1-\theta) k\left(\frac{k}{k+1}\right)^{n-1} \mathbf{M}_{X_{\lfloor(m, n) k+1}}^{(i, j)}\left(t_{1}, t_{2}\right)
\end{aligned}
$$

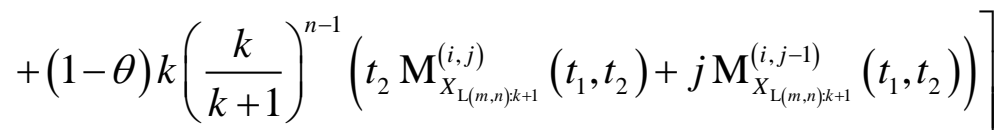

Proof. Similar to the proof of Theorem 5.

By differentiating both sides of equations (15) and (21) with respect to $t_{1}, t_{2}$ and then setting $t_{1}=t_{2}=0$, obtain the recurrence relations for product moments of lower $k$-th record values from the complementary exponential-geometric distribution.

$$
\begin{aligned}
& \mathrm{E}\left(X_{\mathrm{L}(m, n-1) k+1}^{(i, j)}\right)=\frac{1}{k(1-\theta)}\left(\frac{k+1}{k}\right)^{n-1}\left[k \theta ( \frac { k } { k - 1 } ) ^ { n - 1 } \mathrm { E } \left(X_{\mathrm{L}_{(m, n,-1) k-1}^{(i, j)}}^{(i, 1)}\right.\right. \\
& +(1-2 \theta) k \theta \mathrm{E}\left(X_{\llcorner(m, n-1) k}^{(i, j)}\right)-j k \theta\left(\frac{k}{k-1}\right)^{n-1} \mathrm{E}\left(X_{\llcorner(m, n) k-1}^{(i, j-1)}\right) \\
& -k(1-2 \theta) j \mathrm{E}\left(X_{\mathrm{L}(m, n) k}^{(i, j-1)}\right)+k j(1-\theta)\left(\frac{k}{k+1}\right)^{n-1} \mathrm{E}\left(X_{\llcorner(m, n) k+1}^{(i, j-1)}\right) \\
& \left.-j \mathrm{E}\left(X_{\mathrm{L}(m, n) k k}^{(i, j-1)}\right)\right]
\end{aligned}
$$

and 


\section{KUMAR ET AL}

$$
\begin{aligned}
& \mathrm{E}\left(X_{\llcorner(m, n) k}^{(i, j-1)}\right)=\frac{1}{j(1-k(1-2 \theta))}\left[\frac{\theta k^{n}}{(k-1)^{n-1}} \mathrm{E}\left(X_{\llcorner(m, n-1) k-1}^{(i, j)}\right)-\frac{j \theta k^{n}}{(k-1)^{n-2}} \mathrm{E}\left(X_{\llcorner(m, n) k)-1}^{(i, j-1)}\right)\right. \\
& +(1-2 \theta) \mathrm{E}\left(X_{\mathrm{L}(m, n-1))_{k}}^{(i, j)}\right)-(1-\theta) k\left(\frac{k}{k-1}\right)^{n-1} \mathrm{E}\left(X_{L(m, n-1) k-1}^{(i, j)}\right) \\
& \left.+(1-\theta) k\left(\frac{k}{k-1}\right)^{n-1} j \mathrm{E}\left(X_{\lfloor(m, n) k+1}^{(i, j-1)}\right)\right]
\end{aligned}
$$

Remark 4. Setting $k=1$ in (15) and (21), deduce the recurrence relations for joint moment generating function of lower record value from the complementary exponential-geometric distribution.

Remark 5. Equation (6) can be deduced from equation (15) by setting $j=0$.

\section{Numerical Results}

The recurrence relations for the first and second single moments of lower record values allow us to evaluate the means and variances of all lower record values. For the computation of variances and covariances, the product moment were computed first. The diagonal elements are obtained from the recurrence relations. Next, the recurrence relation was used for the computation of the product moment of lower record values. These values were used to evaluate the covariance. When the recurrence relations to calculate the moments is used, only a few initial moments are needed to be numerically calculated. The values of means, variances and covariances were computed using R software. In Tables 1-3, we have computed the values of means for $\theta=0.25,0.5,0.75$ and $\beta=0.5(0.5) 3$. From Tables 1-3, the means are decreasing with respect to $n, \beta$, and $\theta$. In Tables 4-6, we have computed the variances and covariances for different values $m$ and $n$ and for different values of $\beta$ and $\theta$. Variances and covariances decrease for both $\beta$ and $\theta$ values as $n$ increases. 


\section{MOMENT GENERATING FUNCTIONS OF CEG DISTRIBUTION}

Table 1. Means of lower record values for $\theta=0.25$

\begin{tabular}{rrrrrrr}
$\boldsymbol{n}$ & $\boldsymbol{\beta}=\mathbf{0 . 5}$ & $\boldsymbol{\beta}=\mathbf{1 . 0}$ & $\boldsymbol{\beta}=\mathbf{1 . 5}$ & $\boldsymbol{\beta}=\mathbf{2 . 0}$ & $\boldsymbol{\beta}=\mathbf{2 . 5}$ & $\boldsymbol{\beta}=\mathbf{3 . 0}$ \\
\hline 1 & 7.498627 & 4.857795 & 3.476042 & 2.653920 & 2.120225 & 1.751189 \\
2 & 2.278825 & 1.525475 & 1.120836 & 0.874487 & 0.711305 & 0.596444 \\
3 & 0.779095 & 0.537407 & 0.404214 & 0.321322 & 0.265365 & 0.225329 \\
4 & 0.295641 & 0.209053 & 0.160229 & 0.129257 & 0.108009 & 0.092597 \\
5 & 0.122002 & 0.087910 & 0.068325 & 0.055711 & 0.046949 & 0.040525 \\
6 & 0.053580 & 0.039127 & 0.030709 & 0.025225 & 0.021381 & 0.018542 \\
7 & 0.024580 & 0.018114 & 0.014310 & 0.011813 & 0.010052 & 0.008744 \\
8 & 0.011613 & 0.008610 & 0.006831 & 0.005658 & 0.004826 & 0.004207 \\
9 & 0.005595 & 0.004164 & 0.003314 & 0.002750 & 0.002350 & 0.002051 \\
10 & 0.002730 & 0.002037 & 0.001624 & 0.001350 & 0.001155 & 0.001009 \\
\hline
\end{tabular}

Table 2. Means of lower record values for $\theta=0.5$

\begin{tabular}{rrrrrrr}
$\boldsymbol{n}$ & $\boldsymbol{\beta}=\mathbf{0 . 5}$ & $\boldsymbol{\beta}=\mathbf{1 . 0}$ & $\boldsymbol{\beta}=\mathbf{1 . 5}$ & $\boldsymbol{\beta}=\mathbf{2 . 0}$ & $\boldsymbol{\beta}=\mathbf{2 . 5}$ & $\boldsymbol{\beta}=\mathbf{3 . 0}$ \\
\hline 1 & 2.293903 & 1.583854 & 1.191708 & 0.947316 & 0.782197 & 0.663994 \\
2 & 0.787023 & 0.555218 & 0.424690 & 0.342004 & 0.285360 & 0.244326 \\
3 & 0.298723 & 0.214544 & 0.166323 & 0.135340 & 0.113863 & 0.098148 \\
4 & 0.123094 & 0.089625 & 0.070185 & 0.057553 & 0.048715 & 0.042199 \\
5 & 0.053952 & 0.039670 & 0.031288 & 0.025796 & 0.021927 & 0.019059 \\
6 & 0.024704 & 0.018287 & 0.014493 & 0.011993 & 0.010224 & 0.008907 \\
7 & 0.011654 & 0.008666 & 0.006890 & 0.005715 & 0.004881 & 0.004259 \\
8 & 0.005608 & 0.004183 & 0.003333 & 0.002769 & 0.002368 & 0.002068 \\
9 & 0.002735 & 0.002043 & 0.001630 & 0.001356 & 0.001160 & 0.001014 \\
10 & 0.001345 & 0.001006 & 0.000804 & 0.000669 & 0.000573 & 0.000501 \\
\hline
\end{tabular}

Table 3. Means of lower record values for $\theta=0.75$

\begin{tabular}{rrrrrrr}
$\boldsymbol{n}$ & $\boldsymbol{\beta}=\mathbf{0 . 5}$ & $\boldsymbol{\beta}=\mathbf{1 . 0}$ & $\boldsymbol{\beta}=\mathbf{1 . 5}$ & $\boldsymbol{\beta}=\mathbf{2 . 0}$ & $\boldsymbol{\beta}=\mathbf{2 . 5}$ & $\boldsymbol{\beta}=\mathbf{3 . 0}$ \\
\hline 1 & 1.284651 & 0.909454 & 0.697410 & 0.562694 & 0.470189 & 0.403047 \\
2 & 0.461991 & 0.332279 & 0.257850 & 0.209968 & 0.176743 & 0.152413 \\
3 & 0.182181 & 0.132703 & 0.103947 & 0.085254 & 0.072172 & 0.062523 \\
4 & 0.077252 & 0.056804 & 0.044802 & 0.036937 & 0.031398 & 0.027290 \\
5 & 0.034552 & 0.025575 & 0.020268 & 0.016771 & 0.014296 & 0.012454 \\
6 & 0.016040 & 0.011926 & 0.009482 & 0.007865 & 0.006717 & 0.005860 \\
7 & 0.007636 & 0.005695 & 0.004537 & 0.003769 & 0.003223 & 0.002815 \\
8 & 0.003697 & 0.002762 & 0.002204 & 0.001833 & 0.001569 & 0.001371 \\
9 & 0.001810 & 0.001354 & 0.001081 & 0.000900 & 0.000770 & 0.000674 \\
10 & 0.000892 & 0.000668 & 0.000534 & 0.000445 & 0.000381 & 0.000333 \\
\hline
\end{tabular}




\section{KUMAR ET AL}

Table 4. Variances and covariances of lower record values for $\theta=0.25$

\begin{tabular}{|c|c|c|c|c|c|c|c|}
\hline$m$ & $n$ & 0.2 & 0.4 & 0.6 & 0.8 & 1.0 & 1.2 \\
\hline 1 & 1 & 8.8540090 & 5.4945480 & 3.6235200 & 2.5069460 & 1.8030190 & 1.3389290 \\
\hline 1 & 2 & 2.4780752 & 1.5627858 & 1.0459983 & 0.7336188 & 0.5343071 & 0.4014186 \\
\hline 1 & 3 & 0.7809840 & 0.5004903 & 0.3398846 & 0.2415405 & 0.1780381 & 0.1352275 \\
\hline 1 & 4 & 0.2755835 & 0.1791941 & 0.1232751 & 0.0886235 & 0.0660041 & 0.0506030 \\
\hline 1 & 5 & 0.1072334 & 0.0705655 & 0.0490553 & 0.0355927 & 0.0267257 & 0.0206393 \\
\hline 1 & 6 & 0.0450741 & 0.0299302 & 0.0209697 & 0.0153186 & 0.0115712 & 0.0089834 \\
\hline 1 & 7 & 0.0200488 & 0.0133985 & 0.0094389 & 0.0069280 & 0.0052550 & 0.0040946 \\
\hline 1 & 8 & 0.0092756 & 0.0062259 & 0.0044024 & 0.0032417 & 0.0024657 & 0.0019259 \\
\hline 1 & 9 & 0.0044066 & 0.0029664 & 0.0021028 & 0.0015516 & 0.0011824 & 0.0009251 \\
\hline 2 & 2 & 1.2504095 & 0.7888095 & 0.5281692 & 0.3706015 & 0.2700474 & 0.2029881 \\
\hline 2 & 3 & 0.3723332 & 0.2387714 & 0.1622890 & 0.1154436 & 0.0851822 & 0.0647705 \\
\hline 2 & 4 & 0.1244129 & 0.0810439 & 0.0558636 & 0.0402439 & 0.0300357 & 0.0230759 \\
\hline 2 & 5 & 0.0461769 & 0.0304912 & 0.0212706 & 0.0154867 & 0.0116681 & 0.0090406 \\
\hline 2 & 6 & 0.0186997 & 0.0124808 & 0.0087881 & 0.0064509 & 0.0048954 & 0.0038173 \\
\hline 2 & 7 & 0.0080933 & 0.0054445 & 0.0038597 & 0.0028498 & 0.0021738 & 0.0017028 \\
\hline 2 & 8 & 0.0036737 & 0.0024849 & 0.0017699 & 0.0013121 & 0.0010044 & 0.0007892 \\
\hline 2 & 9 & 0.0017229 & 0.0011697 & 0.0008358 & 0.0006213 & 0.0004767 & 0.0003753 \\
\hline 3 & 3 & 0.1857155 & 0.1191201 & 0.0809868 & 0.0576293 & 0.0425391 & 0.0323591 \\
\hline 3 & 4 & 0.0588114 & 0.0383443 & 0.0264577 & 0.0190809 & 0.0142571 & 0.0109662 \\
\hline 3 & 5 & 0.0207815 & 0.0137528 & 0.0096159 & 0.0070173 & 0.0052991 & 0.0041149 \\
\hline 3 & 6 & 0.0169659 & 0.0114037 & 0.0080833 & 0.0059707 & 0.0045576 & 0.0035735 \\
\hline 3 & 7 & 0.0071707 & 0.0048643 & 0.0034755 & 0.0025849 & 0.0019851 & 0.0015647 \\
\hline 3 & 8 & 0.0032000 & 0.0021851 & 0.0015699 & 0.0011733 & 0.0009048 & 0.0007159 \\
\hline 3 & 9 & 0.0014833 & 0.0010174 & 0.0007338 & 0.0005502 & 0.0004255 & 0.0003375 \\
\hline 4 & 4 & 0.0637823 & 0.0417816 & 0.0289564 & 0.0209686 & 0.0157274 & 0.0121401 \\
\hline 4 & 5 & 0.0218023 & 0.0145100 & 0.0101991 & 0.0074796 & 0.0056742 & 0.0044252 \\
\hline 4 & 6 & 0.0082352 & 0.0055556 & 0.0039518 & 0.0029287 & 0.0022426 & 0.0017635 \\
\hline 4 & 7 & 0.0033769 & 0.0023025 & 0.0016530 & 0.0012349 & 0.0009523 & 0.0007535 \\
\hline 4 & 8 & 0.0014739 & 0.0010128 & 0.0007320 & 0.0005500 & 0.0004263 & 0.0003388 \\
\hline 4 & 9 & 0.0006727 & 0.0004648 & 0.0003375 & 0.0002546 & 0.0001980 & 0.0001578 \\
\hline 5 & 5 & 0.0114207 & 0.0076101 & 0.0053555 & 0.0039320 & 0.0029861 & 0.0023312 \\
\hline 5 & 6 & 0.0041464 & 0.0028039 & 0.0019990 & 0.0014847 & 0.0011391 & 0.0008975 \\
\hline 5 & 7 & 0.0016460 & 0.0011265 & 0.0008115 & 0.0006083 & 0.0004705 & 0.0003733 \\
\hline 5 & 8 & 0.0007010 & 0.0004841 & 0.0003515 & 0.0002652 & 0.0002063 & 0.0001646 \\
\hline 5 & 9 & 0.0003144 & 0.0002185 & 0.0001595 & 0.0001209 & 0.0000945 & 0.0000756 \\
\hline 6 & 6 & 0.0021597 & 0.0014622 & 0.0010436 & 0.0007759 & 0.0005960 & 0.0004700 \\
\hline 6 & 7 & 0.0008284 & 0.0005683 & 0.0004104 & 0.0003082 & 0.0002388 & 0.0001899 \\
\hline 6 & 8 & 0.0003435 & 0.0002381 & 0.0001734 & 0.0001313 & 0.0001024 & 0.0000819 \\
\hline 6 & 9 & 0.0001510 & 0.0001055 & 0.0000773 & 0.0000589 & 0.0000461 & 0.0000370 \\
\hline 7 & 7 & 0.0004296 & 0.0002950 & 0.0002133 & 0.0001603 & 0.0001244 & 0.0000989 \\
\hline 7 & 8 & 0.0001730 & 0.0001202 & 0.0000877 & 0.0000665 & 0.0000520 & 0.0000417 \\
\hline 7 & 9 & 0.0000246 & 0.0000171 & 0.0000125 & 0.0000095 & 0.0000075 & 0.0000060 \\
\hline 8 & 8 & 0.0000277 & 0.0000192 & 0.0000139 & 0.0000105 & 0.0000082 & 0.0000065 \\
\hline 8 & 9 & 0.0000114 & 0.0000079 & 0.0000058 & 0.0000044 & 0.0000035 & 0.0000028 \\
\hline
\end{tabular}




\section{MOMENT GENERATING FUNCTIONS OF CEG DISTRIBUTION}

Table 5. Variances and covariances of lower record values for $\theta=0.5$

\begin{tabular}{|c|c|c|c|c|c|c|c|}
\hline$m$ & $n$ & 0.2 & 0.4 & 0.6 & 0.8 & 1.0 & 1.2 \\
\hline 1 & 1 & 6.3262050 & 4.2259140 & 2.9791380 & 2.1898860 & 1.6643720 & 1.2998970 \\
\hline 1 & 2 & 2.0784553 & 1.4052046 & 1.0012573 & 0.7430432 & 0.5695783 & 0.4482855 \\
\hline 1 & 3 & 0.7592399 & 0.5187581 & 0.3730687 & 0.2791262 & 0.2155187 & 0.1707236 \\
\hline 1 & 4 & 0.3033588 & 0.2090349 & 0.1514347 & 0.1140292 & 0.0885412 & 0.0704888 \\
\hline 1 & 5 & 0.1299449 & 0.0901056 & 0.0656297 & 0.0496502 & 0.0387101 & 0.0309287 \\
\hline 1 & 6 & 0.0585454 & 0.0407759 & 0.0298118 & 0.0226266 & 0.0176909 & 0.0141698 \\
\hline 1 & 7 & 0.0273160 & 0.0190823 & 0.0139869 & 0.0106390 & 0.0083340 & 0.0066864 \\
\hline 1 & 8 & 0.0130487 & 0.0091337 & 0.0067061 & 0.0051083 & 0.0040066 & 0.0032181 \\
\hline 1 & 9 & 0.0063313 & 0.0044376 & 0.0032617 & 0.0024870 & 0.0019522 & 0.0015691 \\
\hline 2 & 2 & 1.5427515 & 1.0494398 & 0.7519190 & 0.5608180 & 0.4318625 & 0.3413155 \\
\hline 2 & 3 & 0.5516305 & 0.3795269 & 0.2746518 & 0.2066582 & 0.1603867 & 0.1276464 \\
\hline 2 & 4 & 0.2164982 & 0.1503447 & 0.1096799 & 0.0831099 & 0.0649019 & 0.0519374 \\
\hline 2 & 5 & 0.0914716 & 0.0639695 & 0.0469494 & 0.0357622 & 0.0280550 & 0.0225412 \\
\hline 2 & 6 & 0.0408051 & 0.0286797 & 0.0211389 & 0.0161610 & 0.0127186 & 0.0102476 \\
\hline 2 & 7 & 0.0189087 & 0.0133355 & 0.0098577 & 0.0075552 & 0.0059587 & 0.0048101 \\
\hline 2 & 8 & 0.0089909 & 0.0063554 & 0.0047071 & 0.0036136 & 0.0028541 & 0.0023068 \\
\hline 2 & 9 & 0.0043491 & 0.0030789 & 0.0022833 & 0.0017548 & 0.0013873 & 0.0011222 \\
\hline 3 & 3 & 0.4140999 & 0.2865114 & 0.2083795 & 0.1574953 & 0.1227234 & 0.0980258 \\
\hline 3 & 4 & 0.1599436 & 0.1117700 & 0.0819941 & 0.0624404 & 0.0489783 & 0.0393519 \\
\hline 3 & 5 & 0.0667386 & 0.0469950 & 0.0347018 & 0.0265764 & 0.0209499 & 0.0169058 \\
\hline 3 & 6 & 0.0295019 & 0.0208887 & 0.0154968 & 0.0119161 & 0.0094262 & 0.0076299 \\
\hline 3 & 7 & 0.0135844 & 0.0096549 & 0.0071857 & 0.0055406 & 0.0043933 & 0.0035635 \\
\hline 3 & 8 & 0.0064316 & 0.0045828 & 0.0034181 & 0.0026404 & 0.0020970 & 0.0017032 \\
\hline 3 & 9 & 0.0031022 & 0.0022142 & 0.0016538 & 0.0012791 & 0.0010169 & 0.0008267 \\
\hline 4 & 4 & 0.1215066 & 0.0853253 & 0.0628627 & 0.0480525 & 0.0378190 & 0.0304770 \\
\hline 4 & 5 & 0.0501210 & 0.0354828 & 0.0263240 & 0.0202435 & 0.0160162 & 0.0129667 \\
\hline 4 & 6 & 0.0219689 & 0.0156447 & 0.0116648 & 0.0090091 & 0.0071545 & 0.0058113 \\
\hline 4 & 7 & 0.0100557 & 0.0071903 & 0.0053797 & 0.0041673 & 0.0033179 & 0.0027010 \\
\hline 4 & 8 & 0.0047416 & 0.0033999 & 0.0025497 & 0.0019790 & 0.0015783 & 0.0012868 \\
\hline 4 & 9 & 0.0022809 & 0.0016384 & 0.0012306 & 0.0009564 & 0.0007636 & 0.0006232 \\
\hline 5 & 5 & 0.0385091 & 0.0273718 & 0.0203772 & 0.0157180 & 0.0124690 & 0.0101188 \\
\hline 5 & 6 & 0.0167432 & 0.0119750 & 0.0089621 & 0.0069444 & 0.0055307 & 0.0045038 \\
\hline 5 & 7 & 0.0076200 & 0.0054736 & 0.0041115 & 0.0031959 & 0.0025522 & 0.0020832 \\
\hline 5 & 8 & 0.0035790 & 0.0025785 & 0.0019416 & 0.0015124 & 0.0012100 & 0.0009892 \\
\hline 5 & 9 & 0.0017171 & 0.0012395 & 0.0009349 & 0.0007292 & 0.0005841 & 0.0004781 \\
\hline 6 & 6 & 0.0129911 & 0.0093207 & 0.0069945 & 0.0054325 & 0.0043354 & 0.0035368 \\
\hline 6 & 7 & 0.0058790 & 0.0042372 & 0.0031919 & 0.0024873 & 0.0019906 & 0.0016280 \\
\hline 6 & 8 & 0.0027505 & 0.0019886 & 0.0015019 & 0.0011729 & 0.0009406 & 0.0007705 \\
\hline 6 & 9 & 0.0013161 & 0.0009535 & 0.0007214 & 0.0005642 & 0.0004530 & 0.0003715 \\
\hline 7 & 7 & 0.0045980 & 0.0033218 & 0.0025075 & 0.0019573 & 0.0015689 & 0.0012848 \\
\hline 7 & 8 & 0.0021427 & 0.0015530 & 0.0011755 & 0.0009197 & 0.0007387 & 0.0006060 \\
\hline 7 & 9 & 0.0010225 & 0.0007427 & 0.0005632 & 0.0004413 & 0.0003549 & 0.0002915 \\
\hline 8 & 8 & 0.0016862 & 0.0012243 & 0.0009280 & 0.0007270 & 0.0005846 & 0.0004800 \\
\hline 8 & 9 & 0.0008024 & 0.0005839 & 0.0004435 & 0.0003480 & 0.0002802 & 0.0002304 \\
\hline
\end{tabular}




\section{KUMAR ET AL}

Table 6. Variances and covariances of lower record values for $\theta=0.6$

\begin{tabular}{|c|c|c|c|c|c|c|c|}
\hline$m$ & $n$ & 0.2 & 0.4 & 0.6 & 0.8 & 1.0 & 1.2 \\
\hline 1 & 1 & 5.0738580 & 3.4420660 & 2.4600120 & 1.8305140 & 1.4065540 & 1.1094200 \\
\hline 1 & 2 & 1.7295697 & 1.1860306 & 0.8557216 & 0.6421224 & 0.4971114 & 0.3947366 \\
\hline 1 & 3 & 0.6522793 & 0.4514078 & 0.3282963 & 0.2480763 & 0.1932415 & 0.1542892 \\
\hline 1 & 4 & 0.2672931 & 0.1863044 & 0.1363294 & 0.1035690 & 0.0810548 & 0.0649846 \\
\hline 1 & 5 & 0.1166466 & 0.0817262 & 0.0600696 & 0.0458099 & 0.0359716 & 0.0289246 \\
\hline 1 & 6 & 0.0532419 & 0.0374374 & 0.0276012 & 0.0211045 & 0.0166100 & 0.0133828 \\
\hline 1 & 7 & 0.0250609 & 0.0176645 & 0.0130501 & 0.0099960 & 0.0078792 & 0.0063568 \\
\hline 1 & 8 & 0.0250609 & 0.0176645 & 0.0130501 & 0.0099960 & 0.0078792 & 0.0063568 \\
\hline 1 & 9 & 0.0120416 & 0.0085013 & 0.0062891 & 0.0048229 & 0.0038054 & 0.0030728 \\
\hline 2 & 2 & 1.4711976 & 1.0164525 & 0.7382938 & 0.5573363 & 0.4338054 & 0.3461500 \\
\hline 2 & 3 & 0.5497578 & 0.3835629 & 0.2809775 & 0.2136950 & 0.1674271 & 0.1343784 \\
\hline 2 & 4 & 0.2236305 & 0.1572308 & 0.1159433 & 0.0886876 & 0.0698357 & 0.0562997 \\
\hline 2 & 5 & 0.0970613 & 0.0686276 & 0.0508504 & 0.0390581 & 0.0308668 & 0.0249629 \\
\hline 2 & 6 & 0.0441328 & 0.0313270 & 0.0232894 & 0.0179397 & 0.0142126 & 0.0115191 \\
\hline 2 & 7 & 0.0207192 & 0.0147462 & 0.0109873 & 0.0084797 & 0.0067291 & 0.0054618 \\
\hline 2 & 8 & 0.0099381 & 0.0070855 & 0.0052872 & 0.0040857 & 0.0032458 & 0.0026370 \\
\hline 2 & 9 & 0.0048350 & 0.0034512 & 0.0025778 & 0.0019937 & 0.0015850 & 0.0012885 \\
\hline 3 & 3 & 0.4788187 & 0.3362737 & 0.2477615 & 0.1893954 & 0.1490620 & 0.1201233 \\
\hline 3 & 4 & 0.1938386 & 0.1372307 & 0.1018093 & 0.0782924 & 0.0619422 & 0.0501472 \\
\hline 3 & 5 & 0.0838327 & 0.0597019 & 0.0445150 & 0.0343809 & 0.0273035 & 0.0221773 \\
\hline 3 & 6 & 0.0380233 & 0.0271903 & 0.0203445 & 0.0157600 & 0.0125482 & 0.0102153 \\
\hline 3 & 7 & 0.0178209 & 0.0127792 & 0.0095842 & 0.0074394 & 0.0059335 & 0.0048377 \\
\hline 3 & 8 & 0.0085384 & 0.0061341 & 0.0046077 & 0.0035813 & 0.0028596 & 0.0023339 \\
\hline 3 & 9 & 0.0041510 & 0.0029858 & 0.0022451 & 0.0017465 & 0.0013956 & 0.0011398 \\
\hline 4 & 4 & 0.1726281 & 0.1228683 & 0.0915759 & 0.0707071 & 0.0561396 & 0.0455924 \\
\hline 4 & 5 & 0.0744937 & 0.0533417 & 0.0399609 & 0.0309907 & 0.0247002 & 0.0201271 \\
\hline 4 & 6 & 0.0337354 & 0.0242584 & 0.0182380 & 0.0141872 & 0.0113373 & 0.0092595 \\
\hline 4 & 7 & 0.0157948 & 0.0113901 & 0.0085839 & 0.0066910 & 0.0053564 & 0.0043815 \\
\hline 4 & 8 & 0.0075624 & 0.0054638 & 0.0041242 & 0.0032191 & 0.0025800 & 0.0021126 \\
\hline 4 & 9 & 0.0036749 & 0.0026584 & 0.0020087 & 0.0015693 & 0.0012587 & 0.0010313 \\
\hline 5 & 5 & 0.0676009 & 0.0486023 & 0.0365367 & 0.0284203 & 0.0227109 & 0.0185488 \\
\hline 5 & 6 & 0.0305842 & 0.0220820 & 0.0166596 & 0.0129985 & 0.0104147 & 0.0085257 \\
\hline 5 & 7 & 0.0143102 & 0.0103616 & 0.0078361 & 0.0061266 & 0.0049175 & 0.0040318 \\
\hline 5 & 8 & 0.0068487 & 0.0049684 & 0.0037634 & 0.0029464 & 0.0023676 & 0.0019432 \\
\hline 5 & 9 & 0.0033271 & 0.0024167 & 0.0018325 & 0.0014359 & 0.0011548 & 0.0009484 \\
\hline 6 & 6 & 0.0281612 & 0.0203920 & 0.0154228 & 0.0120591 & 0.0096800 & 0.0079372 \\
\hline 6 & 7 & 0.0131705 & 0.0095641 & 0.0072508 & 0.0056810 & 0.0045683 & 0.0037516 \\
\hline 6 & 8 & 0.0063013 & 0.0045845 & 0.0034812 & 0.0027312 & 0.0021988 & 0.0018075 \\
\hline 6 & 9 & 0.0030606 & 0.0022295 & 0.0016947 & 0.0013308 & 0.0010722 & 0.0008820 \\
\hline 7 & 7 & 0.0122564 & 0.0089184 & 0.0067730 & 0.0053145 & 0.0042790 & 0.0035180 \\
\hline 7 & 8 & 0.0058625 & 0.0042738 & 0.0032508 & 0.0025542 & 0.0020589 & 0.0016944 \\
\hline 7 & 9 & 0.0028470 & 0.0020780 & 0.0015822 & 0.0012443 & 0.0010038 & 0.0008266 \\
\hline 8 & 8 & 0.0054964 & 0.0040125 & 0.0030556 & 0.0024033 & 0.0019389 & 0.0015969 \\
\hline 8 & 9 & 0.0026687 & 0.0019506 & 0.0014869 & 0.0011705 & 0.0009451 & 0.0007788 \\
\hline
\end{tabular}




\section{MOMENT GENERATING FUNCTIONS OF CEG DISTRIBUTION}

\section{Conclusion}

The lower record values for complementary exponential-geometric distribution were considered. Explicit expressions were obtained, as well as recurrence relations for the marginal and joint moment generating functions of lower of record values. The recurrence relations obtained in the paper allows us to evaluate the means, variances and covariances of all lower record values for all sample sizes in a simple recursive manner.

\section{Acknowledgments}

The authors would like to thanks the reviewers and the editors for their comments which helped improve the paper.

\section{References}

Ahsanullah, M. (1995). Record statistics. New York, NY: Nova Science Publishers.

Arnold, B. C., Balakrishnan, N., \& Nagaraja, H. N. (1992). A first course in order. New York, NY: John Wiley and Sons.

Arnold, B. C., Balakrishnan, N., \& Nagaraja, H. N. (1998). Records. New York, NY: John Wiley and Sons. doi: 10.1002/9781118150412

Balakrishnan, N., \& Ahsanullah, M. (1993). Relations for single and product moments of record values from exponential distribution. Journal of Applied Statistical Science, 2(1), 73-87.

Balakrishnan, N., \& Ahsanullah, M. (1994). Recurrence relations for single and product moments of record values from generalized Pareto distribution. Communications in Statistics - Theory and Methods, 23(10), 2841-2852. doi: 10.1080/03610929408831419

Chandler, K. N. (1952). The distribution and frequency of record values. Journal of the Royal Statistical Society. Series B (Methodological), 14(2), 220228. Available from http://www.jstor.org/stable/2983870

Glick, N. (1978). Breaking records and breaking boards. The American Mathematical Monthly, 85(1), 2-26. doi: 10.2307/2978044

Grunzień, Z., \& Szynal, D. (1997). Characterization of uniform and exponential distributions via moments of the $k$ th record values with random indices. Journal of Applied Statistical Science, 5(4), 259-266. 


\section{KUMAR ET AL}

Kumar, D. (2012). Recurrence relations for marginal and joint moment generating functions of generalized logistic distribution based on lower $k$ record values and its characterization. ProbStat Forum, 05(April), 47-53. Retrieved from http://probstat.org.in/PSF-2012-06.pdf

Kumar, D. (2015). Explicit expressions and statistical inference of generalized Rayleigh distribution based on lower record values. Mathematical Methods of Statistics, 24(3), 225-241. doi: 10.3103/s1066530715030035

Kumar, D. (2016). $k$-th lower record values from of Dagum distribution and characterization. Discussions Mathematicae Probability and Statistics, 36(1-2), 25-41. doi: 10.7151/dmps.1183

Kumar, D., \& Kulshrestha, A. (2013). Expectation identities of upper record values from generalized Pareto distribution and a characterization. Journal of Statistics Applications \& Probability, 2(2), 115-121. doi: 10.12785/jsap/020204

Louzada, F., Roman, M., \& Cancho, V. G. (2011). The complementary exponential geometric distribution: Model, properties, and a comparison with its counterpart. Computational Statistics \& Data Analysis, 55(8), 2516-2524. doi: 10.1016/j.csda.2011.02.018

Nevzorov, V. B. (1987). Records (B. Seckler, Trans.). Theory of Probability \& Its Applications, 32(2), 201-228. doi: 10.1137/1132032

Pawlas, P., \& Szynal, D. (1999). Recurrence relations for single and product moments of $k$-th record values from Pareto, generalized Pareto and Burr distributions. Communications in Statistics - Theory and Methods, 28(7), 16991709. doi: 10.1080/03610929908832380

Resnick, S. I. (1973). Extreme values, regular variation and point processes. New York, NY: Springer-Verlag.

Shorrock, R. W. (1973). Record values and inter-record times. Journal of Applied Probability, 10(3), 543-555. doi: 10.2307/3212775 